\title{
ELIMINATION OF NEGATIVE PROBABILITIES WITHIN THE CAUSAL STOCHASTIC INTERPRETATION OF QUANTUM MECHANICS
}

\author{
․ CUFARO-PETRONI a . C. DEWDNEY ${ }^{b}$, P. HOLLAND ${ }^{b}$. T. KYPRIANIDIS ${ }^{b}$ and J.P. VIGIER b \\ à Istituto di Fisica, Lniversita di Bari, Via Amendola 273, 70126 Bari, Italt. \\ bInstitut Henri Poincaré, Laboratoire de Physique Théorique, 11, rue Pierre et Marie Curie. 75231 Paris Cedex 05, France
}

Received 31 October 1984

\begin{abstract}
Negative probabilities resulting from the Klein-Gordon equation are eliminated from quantum theory within the stochastic interpretation of quantum theory (SIQM) for spin-zero particles. The assumption of real physical paths in $F_{4}$ implies that only particles (antiparticles) of positive energies move forward in time with positive probability densities.
\end{abstract}

The problem raised by the mathematical existence of negative probabilities associated with certain solutions of second-order wave equations raises as recently stressed by Feymman [1] fundamental questions to interpret quantum mechanics.

In the Copenhagen interpretation (CIQM) it is usually accepted that the only way out of the existence of negative probability solutions is a rejection of the first quantized version of the Klein-Gordon equation in favor of the second quantization formalism given by Pauli and Weisskopf [2].

For the causal stochastic interpretation (SIQM) or the pilot-wave model of Einstein and de Broglie the trouble is apparently greater since (as stressed by John Bell [3] ) the very existence of paths in real space time $\mathrm{F}_{4}$ always implies positive probability distributions... which are also needed in quantum statistics if one accepts their reinterpretation in terms of stochastic distinguishable particle motions correlated by actions at a distance [4].

The aim of the present letter is to show that in the SIQM the very existence of initial time-like drift motions and positive initial particle distributions implies. for all spin zero particle systems, the choice of particular positive energy positive density solutions (recent. ly discussed by Rizov et al. [5]) which are preserved by the causal laws of motion.

To separate positive probability distributions we first follow Rizov et al.'s presentation [with metric $(++t)]$ of an initial result of Feshbach and Villars [6]

The wave equation of a charged spinless relativistic particle in an external field $V_{\mu}=e A_{\mu}$

$\left(D_{\mu} D^{\mu} \cdot m^{2}\right) \psi=0$.

where $D_{\mu}=\partial_{\mu} \quad i V_{\mu}^{\prime}$ and $D_{\mu}=\partial_{\mu}+i V_{\mu} . V_{\mu}=e A_{\mu}$ and $\partial_{\mu}=\partial / \partial x^{\mu}$ can be deduced from a lagrangian density

$\mathcal{L}=\bar{D}_{\mu} \dot{\psi}^{*} D_{\mu} \dot{\psi} m^{2} \psi^{*} \dot{\psi}$.

which yields also the expression for the conserved current

$j^{\mu}=\partial \mathcal{L} / \partial V_{\mu}=\psi^{*}\left(\mathrm{i}-1 \dddot{\partial}^{\mu} \quad 2 V^{\mu}\right) \dot{\psi}$

and for the energy density

$T_{00}=\partial_{0} \psi^{*} \partial_{0} \psi+\bar{D} \dot{\psi}^{*} \cdot D \psi+\left(m^{2}-V_{0}^{2}\right) \psi^{*} \psi$.

The procedure which produces a Schrödinger form of the Klcin-Gordon equation consists in setting a twocomponent wavefunction

$\Psi=\left(\begin{array}{l}\psi_{1} \\ \psi_{2}\end{array}\right)=\frac{1}{\sqrt{2 m}}\left(\begin{array}{cc}i \dot{j}- & V^{0} \psi+m \psi \\ i & V^{0} \psi-m \psi\end{array}\right)$.

with $\dot{\psi}=\partial_{0} \psi$. The components $\psi_{1}$ and $\psi_{2}$ have the property to reduce in the nonrelativistic limit to the Schrödinger wavefunction and 0 respectively.

If we now define a hamiltonian

$H=(1 / 2 m) D^{2}\left(\begin{array}{cc}1 & 1 \\ -1 & 1\end{array}\right)+m\left(\begin{array}{rr}1 & 0 \\ 0 & -1\end{array}\right)+V^{0}\left(\begin{array}{ll}1 & 0 \\ 0 & 1\end{array}\right)$. 
then the Klein-Gordon equation can be written in the "Schrödinger" form

$\mathrm{i} \dot{\Psi}=H \Psi$.

This hamiltonian is hermitian with respect to the indefinite inner product

$\langle\Phi, \Psi\rangle=\int \Phi^{*} \sigma_{3} \Psi \mathrm{d}^{3} x=\int \phi^{*}\left(\mathrm{i} \vec{\partial}_{0}-2 V^{0}\right) \psi \mathrm{d}^{3} x$.

Further one can show that if $V_{\mu}$ is well-behaved (e.g. a sum of a Coulomb potential and other stationary four potentials), then the integral of the energy density (which is the mean value of the hamiltonian $H$ in any state $\Psi$ ) is positive, i.e.

$\left.\langle\Psi, H \Psi\rangle=\int T_{00} \mathrm{~d}^{3} x\right\rangle 0$.

One then gets (with $H \Psi=E \Psi$ ) that

$E>0$ implies $\left\langle\Psi_{+}, \Psi_{+}\right\rangle>0$,

and

$E<0 \quad$ implies $\quad\left\langle\Psi_{\ldots}, \Psi_{-}\right\rangle<0$.

where $\Psi_{+}, \Psi_{-}$belong to the positive and negative spectrum of $H$ respectively.

The negative energy part of the solutions above can be associated to antiparticle solutions. This can be shown by means of the charge conjugation operation

$\Psi(x) \rightarrow \Psi^{\mathrm{c}}(x)=\sigma_{1} \Psi^{*}(x)=\left(\begin{array}{l}\psi_{2}^{*}(x) \\ \psi_{1}^{*}(x)\end{array}\right)$.

$\Psi(P) \rightarrow \Psi^{\mathrm{c}}(P)=\sigma_{1} \Psi^{*}(-P)$.

This maps every negative energy solution $\Psi$ of the Schrödinger equation with $H=H\left(\boldsymbol{P}, e A^{\mu}\right)$ to a positive energy solution of the same equation with a hamiltonian $H^{\mathrm{c}}=H\left(-P,-e A^{\mu}\right)$, corresponding to particles of opposite charge and opposite momenta with $\langle\Psi, \Psi\rangle\rangle 0$.

This fact implies that the space of solutions of eq. (2) splits in two subsets, $\{E>0,\langle\Psi, \Psi\rangle>0\} ;\{E<0$, $\langle\Psi . \Psi\rangle\langle 0\}$ and consequently the physical states can be defined as superpositions of positive energy solutions only.

In the SIQM we can show that the equations of motion themselves imply that if positive energy and positive probability density states are initially given in a space-like surface $\Sigma_{0}$ (i.e. Cauchy's initial conditions) they are causally transformed into positive energy- positive probability density states on any subsequent surface $\Sigma$. This can be derived as follows. We start from the Klein-Gordon equation (1) and assume $\psi$ $=\exp (P+i S)$ with which it is dccomposed in a Ham. ilton-Jacobi-type and a continuity equation: (real and imaginary part)

$\left(\partial_{\mu} S-V_{\mu}\right)\left(\partial^{\mu} S-V^{\mu}\right)+m^{2}-\square P \quad \partial_{\mu} P \partial^{\mu} P=0$, $\partial_{\mu}\left[\mathrm{e}^{2 P}\left(\partial_{\mu} S-V^{\mu}\right)\right]=0$.

In the stochastic interpretation $P, S$ and $V$ can in general be functions not only defined in quantum configuration space but also associated with the mean trajectories of the quantum particle. In such a case we have

$P=P\left(x^{\mu}(\tau), \tau\right), \quad S=S\left(x^{\mu}(\tau), \tau\right), \quad V=V^{\mu}\left(x^{\mu}(\tau)\right)$,

where $\tau$ denotes the proper time on a trajectory.

However since this model should reproduce the quantum mechanical results, the explicit proper time dependence should vanish, as shown by Guerra and Ruggiero [7] i.c.

$P=P\left(x^{\mu}(\tau)\right), \quad S=S\left(x^{\mu}(\tau)\right)$.

Furthermore if we define the variable mass of de Broglic $M$ as

$M^{2}=m^{2}-\square P-\partial_{\mu} P \partial^{\mu} P$

and calculate the total proper time derivative of the scalar density $\rho=\mathrm{e}^{2 P} M$ along a line of flow we find [8] $\mathrm{d} \rho / \mathrm{d} \tau=\partial_{\mu}\left(\rho u^{\mu}\right)$,

where $u^{\mu}$ is the unit four velocity along a line of flow. Noticing that in the case of presence of an interaction $V^{\mu}$ the four-momentum $P_{\mu}$ is [7]

$P_{\mu}=\partial_{\mu} S-V_{\mu}$

and the unit vector in this direction can be written using the $(\mathrm{HJ})$ equation as

$u_{\mu}=\left(\partial_{\mu} S-V_{\mu}\right) /\left|\partial_{\mu} S-V_{\mu}\right|=\left(\partial_{\mu} S-V_{\mu}\right) / M$.

Now we can write eq. (3) in a form

$\mathrm{d} \rho / \mathrm{d} \tau=\partial_{\mu}\left[\rho\left(\partial_{\mu} S-V_{\mu}\right) / M\right]=\partial_{\mu}\left[\mathrm{e}^{2 P}\left(\partial_{\mu} S-V_{\mu}\right)\right]=0$

and $\mathrm{d} \rho / \mathrm{d} t$ vanishes identically because of the continuity equation. As a consequence we see that $\rho=$ $\mathrm{e}^{2 P} M$ is constant along a line of flow i.e. $=K$, where $K$ is real or purely imaginary. 
For the determination of this constant we can pro. ceed as follows: Noting that $P_{\mu}=\alpha_{\mu} S-V_{\mu}$ we can perform a transformation to the rest trame where this expression reduces to $P_{0}=\partial_{0} S-V_{0}$ i.e. the energy $E$ of the particle. Since the energy $E$ in the rest frame is numerically equal to the variable mass $M$, we realize that $P_{0}$ is an expression for the variable mass. Now if the initial conditions on the quantum particle imply a time-like trajectory (with initially $E>0$ ) then $M$ is real and initially also positive. With this information we can also deduce the positive character of the real constant $K$, since $\mathrm{e}^{2 P}>0$ and $M$ was shown to be positive. Due to the fact that $\mathrm{c}^{2 P} M=K$ all along a line of flow, and since $\mathrm{e}^{2 P}>0$ is always true, we conclude that if $P_{\mu}$ of a particle was initially time-like and $E>0$ then it remains always time-like and $M$ remains positive along the trajectory.

A final step consists in associating this property with the density as the fourth component of the conserved four current

$j_{\mu}=\psi\left(x^{\mu}(\tau)\right)^{*}\left(\mathrm{i}^{-1} \vec{\partial}_{\mu}-2 V_{\mu}\right) \psi\left(x^{\mu}(\tau)\right)$.

In fact this reduces in the rest frame of the particle to $j_{0}=2 \mathrm{e}^{2 P}\left(\partial_{0} S \cdot V_{0}\right)$.

Lising the same argument as above we realize that this is numerically equal to $2 \mathrm{e}^{2 P} M$ which is a conserved positive quantity if the initial condition was $E>0$. Thus positive energy solutions imply positive densities and this character, once established, is conserved along any time-like trajectory. We have thus shown that local positive energy solutions are always associated with local positive densities, a solution that is compatible with the solutions of Rizov et al.. but does not only concern the integrated values but the local values instead. Of course. by the same procedures one proves that $E<0$ is associated with $j_{0}<0$ solutions and can be transformed by means of the charge conjugation operation to $E>0, j_{0}>0$ antiparticle solutions.

As we shall show in subsequent publications this reasoning can be extended

(i) to the case of the Proca spin one equation and

(ii) to the Feynman-Gell-Mann 2-component spinor second-order equation representating spin $1 / 2$ and (iii) to the correlated system of $N$ non-interacting scalar particles in an external field satisfying the causal system [7]

$\left(D_{k}^{2} \quad m_{k}^{2}\right) \psi\left(x_{1} \ldots x_{i v}\right)=0$.

() $r$

$\left(\ddot{D}_{k}^{2} \quad m_{k}^{2}\right) \psi^{*}\left(x_{1} \ldots x_{i}\right)=0$.

where each correlated particle follows real time-like motions in space-time. Indecd all such systems can be treated with the same Foldy Wouthuysen like separation given above and analysed within the SIQM with time-like average causal drift motions influenced by a non-local stochastic quantum potential $[7,9]$.

The authors (C.D., P.H. and T.K.) want to thank the British Royal Society, the Leverhulme Trust and the French Government for grants which made this research possible. Another one (J.P.V.) wants to thank Dr. John Bell for very helpful discussions on the negative probability problem.

\section{References}

[1] R.P. Jeynman, Int. J. Theor. Phys. 21 (1982) 467.

12] W. Pauli and V.F. Weisskopf, Helv. Phys. Acta 1 (1934) 709.

[3] J. Bell, Private communication.

14) A. Kyprianidis, D. Sardelis and J.P. Vigier, Phy's. Lett. 100A (1984) 228 .

15] V.A. Rizov, H. Sazdjian and I.T. Todorov, On the relativistic quantum mechanics of two interacting spinless particles, Orsay preprint IPNO-TH/84-39.

[6] H. Feshbach and F.M.H. Villars, Rev. Mod. Phys. 30 (1958) 24.

17] W. Lehr and J. Park, J. Math. Phys. 18 (1977) 1235; I. Guerra and P. Ruggiero, Lett. Nuovo Cimento 23 (1978) 529;

J.P. Vigier, Lett. Nuovo Cimento 29 (1979) 265; A. Kyprianidis and D. Sardelis, Lett. Nuovo Cimento 39 (1984) 337 .

[8] H. Halbwachs, Théoric relativiste des fluides á spin (Gauthier-Villars, Paris, 1960).

[9] J.P. Vigier, Relativistic predictive quantum potential: , $V$ body case (Bari, 1983) Conf. Proc. (Reidel, Dordrecht), to be published. 